\title{
Intake of $n-3$ fatty acids and long-term outcome in renal transplant recipients: a post hoc analysis of a prospective cohort study
}

\author{
Ilse G. Pranger ${ }^{1 *}$, Eke G. Gruppen ${ }^{1}$, Else van den Berg ${ }^{1}$, Sabita S. Soedamah-Muthu ${ }^{3}$, Gerjan Navis ${ }^{1}$, \\ Rijk O. B. Gans ${ }^{1}$, Frits A. J. Muskiet ${ }^{2}$, Ido P. Kema ${ }^{2}$, Michel M. Joosten ${ }^{1}$ and Stephan J. L. Bakker ${ }^{1}$ \\ ${ }^{1}$ Internal Medicine, University of Groningen and University Medical Center Groningen, PO Box 30.001, \\ 9700 RB Groningen, The Netherlands \\ ${ }^{2}$ Laboratory Medicine, University of Groningen and University Medical Center Groningen, PO Box 30.001, \\ 9700 RB Groningen, The Netherlands \\ ${ }^{3}$ Division of Human Nutrition, Wageningen University, Stippeneng 4, $6708 \mathrm{WE}$ Wageningen, The Netherlands
}

(Submitted 29 April 2016 - Final revision received 24 October 2016 - Accepted 16 November 2016 - First published online 20 December 2016)

\begin{abstract}
Supplementation with $n$-3 fatty acids may improve long-term outcomes of renal transplant recipients (RTR). Recent evidence suggests that EPA and DHA have different outcomes compared with $\alpha$-linolenic acid (ALA). We examined the prospective associations of EPA-DHA and ALA intakes with graft failure and all-cause mortality in 637 RTR. During $3 \cdot 1$ years (interquartile range $2 \cdot 7,3 \cdot 8$ ) of follow-up, forty-one developed graft failure and sixty-seven died. In age- and sex-adjusted analyses, EPA-DHA and ALA intakes were not associated with graft failure. EPA-DHA intake was not significantly associated with mortality (hazard ratio (HR) $0.79 ; 95 \%$ CI $0.54,1.15$ per 0.1 energy $\%$ difference). ALA intake was significantly associated with mortality (HR 1.17; $95 \%$ CI 1.04, 1.31 per 0.1 energy $\%$ difference). This association remained following adjustments for BMI, proteinuria and intakes of fat, carbohydrate and protein. RTR in the highest tertile of ALA intake exhibited about 2-fold higher mortality risk (HR 2.21; $95 \%$ CI 1.23, 3.97) compared with the lowest tertile. In conclusion, ALA intake may be associated with increased mortality in RTR. Future RCT are needed to confirm these results.
\end{abstract}

Key words: $\boldsymbol{n}$-3 Fatty acids: Renal transplant recipients: Mortality: Graft failure

The prevalence of end-stage renal disease (ESRD) is increasing worldwide $^{(1)}$. In the USA, the incidence of ESRD in 2012 was as high as 400 cases per million residents per year ${ }^{(2)}$. The preferred treatment for most ESRD patients is renal transplantation. Compared with dialysis, treatment is less expensive, improves quality of life and increases survival ${ }^{(3,4)}$. Although $90 \%$ of allografts last for at least 1 year ${ }^{(5,6)}$, more than half of these grafts do not last for longer than $10-12$ years $^{(6)}$. This is largely due to graft failure and premature mortality ${ }^{(6)}$. Thus, there is a great need for approaches to improve survival.

Many randomised-controlled trials have investigated whether $n-3$ fatty acid supplements reduce cardiovascular risk and improve graft survival in renal transplant recipients (RTR). A meta-analysis from 2007 concluded that it cannot be excluded that RTR may benefit from such supplements ${ }^{(7)}$. In this respect, it is important to realise that the effects of marine-derived $n-3$ fatty acids such as EPA and DHA and plant-derived $n-3$ fatty acids such as $\alpha$-linolenic acid (ALA) may differ. For example, in the general population, it has been found that supplementation with EPA and DHA was protective, whereas supplementation with ALA was not ${ }^{(8)}$. Importantly, in a large Swedish study including more than
2000 men aged 50 years or older, it was suggested that exposure to ALA has an adverse effect on mortality ${ }^{(9)}$. In RTR, it is unclear whether marine-derived and plant-derived $n-3$ fatty acids differ in effects on long-term outcome.

In the present study, we aimed to investigate the association of marine-derived and plant-derived $n-3$ fatty acids with long-term outcome in stable outpatient RTR. For this purpose, we examined EPA-DHA and ALA intakes separately, and analysed whether the intakes of these $n-3$ fatty acids are associated with graft failure and all-cause mortality.

\section{Methods \\ Design and study population}

We performed a post hoc analysis in an existing prospective cohort study on RTR in which baseline FFQ data were available that were suitable to address our research question. The study took place at the University Medical Center Groningen between November 2008 and March $2011^{(10)}$. All RTR ( $\geq 18$ years of age) had a functioning graft for at least 1 year, no alcohol or drug addiction and

Abbreviations: ALA, $\alpha$-linolenic acid; En\%, energy percentage; HR, hazard ratio; IQR, interquartile range; RTR, renal transplant recipients.

* Corresponding author: I. G. Pranger, email I.g.Pranger@umcg.nl 
sufficient knowledge of the Dutch language. In total, 817 RTR were invited for the study, of whom 707 (87\%) provided written consent to participate. In the present study, patients with missing dietary data were excluded from the analyses, resulting in 637 RTR for analysis. The present study was approved by the Institutional Review Board (METc 2008/186). Dietary intake and clinical parameters were assessed once at baseline. Outcome assessors were blinded to dietary intake measurements and other baseline parameters. RTR did not receive dietary recommendation regarding $n-3$ fatty acid intake. Patients were taking standard immunosuppressive and antihypertensive drugs.

\section{Outcome parameters}

The primary long-term outcomes of the study were graft failure and all-cause mortality. Graft failure was defined as return to dialysis or re-transplantation. The patients were followed-up until 30 April 2013, with no loss to follow-up.

\section{Dietary assessment}

Dietary intake was assessed using a semi-quantitative, validated FFQ that was developed at Wageningen University ${ }^{(11)}$. The questionnaire consisted of 177 food items to record intake during the previous month, expressed in times per day, week or month. The number of servings was recorded in natural units (e.g. slice of bread or apple) or household measures (e.g. cup or spoon). Patients had to fill in the questionnaire at home. All FFQ were checked by a trained researcher and verified with the patients in case of inconsistent answers. The results of the FFQ were converted into total energy and nutrient intake by using the Dutch Food Composition Table of $2006^{(12)}$.

\section{Clinical parameters}

All measurements were obtained during a morning visit to the outpatient clinic. Patients were instructed to collect a 24-h urine sample the day before the visit. In the morning, blood was drawn after a fasting period of $8-12 \mathrm{~h}$. Body weight and height were measured with patients wearing indoor clothing without shoes. BMI was calculated as weight in kilograms divided by height in metres squared $\left(\mathrm{kg} / \mathrm{m}^{2}\right)$, and body surface area was measured as metres squared $\left(\mathrm{m}^{2}\right)$. Diabetes was defined as use of antidiabetic medication or fasting serum glucose $\geq 7.0 \mathrm{mmol} / \mathrm{l}^{(13)}$. Fasting blood pressure, heart rate and mean arterial pressure were determined using a semi-automatic device (Dinamap 1846; Critikon). Blood pressure and heart rate were measured every minute for $15 \mathrm{~min}$, and the last three measures were averaged ${ }^{(14)}$. Renal function was assessed by estimated glomerular filtration rate $(\mathrm{eGFR})^{(15)}$. Serum creatinine levels were measured with a modified version of the Jaffé method (MEGA AU 510; Merck Diagnostica). Total urinary albumin concentration was analysed by nephelometry (Dade Behring Diagnostics), whereas total urinary protein concentration was determined using the Biuret reaction (MEGA AU 150). Serum cholesterol, glycated $\mathrm{Hb}$ and high-sensitive C-reactive protein were measured according to routine laboratory methods. Proteinuria was defined as urinary protein excretion $\geq 0.5 \mathrm{~g} / 24 \mathrm{~h}$.
Information on patient health status, medical history and medication use was obtained from patient records. Information on smoking habits and alcohol intake was collected as well. Patients were divided into current smokers, former smokers or never smokers. In addition, patients were also divided into four groups according to their alcohol intake ( $\mathrm{g}$ ethanol/d) (no alcohol, 0-10, 10-30 and >30 g/d). Smoking behaviour and alcohol intake were based on self-report.

\section{Statistical analyses}

Data analysis was performed using SPSS version 22.0 software (SPSS, Inc.) and $\mathrm{R}$ version 3.2.3. Data are presented as mean values and standard deviations or as medians and interquartile ranges (IQR) unless stated otherwise. n-3 Fatty acid intake was measured as a percentage of total energy intake (En\%). For initial exploration, EPA-DHA and ALA intakes were divided into tertiles, which were compared by means of ANOVA, Kruskal-Wallis or $\chi^{2}$ tests depending on whether the distribution of data was normal, skewed or categorical, respectively. Linear regression analyses with intake of $n-3$ fatty acids as a dependent variable were carried out to compare strengths of associations of different baseline variables with $n-3$ fatty acids. For these analyses, categorical variables were divided into two or more dummy variables. Baseline characteristics with a $P$-value $<0 \cdot 10$ for the association with intake of $n$ - 3 fatty acids were selected as potential explanatory variables in subsequent Cox regression analyses. Cox regression analyses were also used to test whether there were interactions between $n-3$ fatty acid intake and potential explanatory variables (age, sex, BMI, smoking habits, alcohol intake, intakes of protein, fat and carbohydrates, proteinuria, blood pressure). In prospective analyses, we investigated the associations of EPA-DHA and ALA intake with graft failure and all-cause mortality in RTR. Analyses were performed with adjustment for age and sex (model 1) and further cumulative adjustments for BMI (model 2), alcohol intake, smoking behaviour (model 3), total fat intake, total protein intake, total carbohydrate intake (model 4), proteinuria and diastolic blood pressure (DBP) (model 5). Analyses were each time initially performed for EPA-DHA and ALA intakes as continuous variables and repeated for EPA-DHA and ALA intake as tertiles. Cox regression analyses with restricted cubic splines were carried out to show the association between ALA intake and all-cause mortality. A two-sided $P$-value $<0.05$ was considered statistically significant. As there were only a small number of statistical tests related to our a priori hypotheses, correction for multiple testing was not required ${ }^{(16,17)}$

In secondary analyses, $n-3$ fatty acid intake was measured in grams per day instead of En\%. For this analysis, $n-3$ fatty acid intake was adjusted for total energy intake according to the residual method, which is based on the isoenergetic principle $^{(18)}$. In addition, we calculated both the intake of EPA-DHA and the intake of ALA as a proportion of total intake of fatty acids. As sensitivity analyses, we repeated the Cox regression analyses after elimination of patients with extreme intakes of $n-3$ fatty acids outside of $3 \mathrm{SD}$ from the mean value - that is, an EPA-DHA intake $>0.30 \mathrm{En} \%$ or an ALA intake $>1 \cdot 10 \mathrm{En} \%$. 


\section{Results}

Population characteristics of RTR are presented in Table 1. Median time after transplantation was 5.6 (IQR 1.8-12.1) years. The mean age of the study population was 53 (SD 13) years, and $57 \%$ was male. The participants were slightly overweight (mean BMI of $26.6 \mathrm{~kg} / \mathrm{m}^{2}$ ) and $24 \%$ had diabetes. The mean blood pressure of RTR was within normal boundaries $(136 / 83 \mathrm{mmHg})$. It should, however, be noted that $88 \%$ of the 637 participants were using antihypertensive drugs. Mean serum total cholesterol was $5 \cdot 1$ (SD 1.1$) \mathrm{mmol} / \mathrm{l}$, whereas statins were taken by $53 \%$ of the recipients. Calcineurin inhibitors were used by $57 \cdot 1 \%$ of the RTR and $83.3 \%$ were using proliferation inhibitors. Mean eGFR was $49 \mathrm{ml} / \mathrm{min}$ per $1.73 \mathrm{~m}^{2}$. The median urinary protein excretion was 0.19 (IQR $0-8.91) \mathrm{mg} / 24 \mathrm{~h}$, with 139 (22\%) RTR having proteinuria.

Energy intake was 9117 (sD 2664) kJ/d (2175 (sD 637) kcal/d). Of the total intake, $36 \mathrm{En} \%$ was derived from fat (SFA: $13 \mathrm{En} \%$; MUFA: 12 En\%; PUFA: 8 En\%), 15 En\% from proteins and 46 En\% from carbohydrates. Median intakes of EPA-DHA and ALA were $92($ IQR $28-205) \mathrm{mg} / \mathrm{d} \quad(0.04 \quad$ (IQR $0.01-0.09) \quad \mathrm{En} \%)$ and $1.2($ IQR $0.9-1.7) \mathrm{g} / \mathrm{d}(0.5$ (IQR 0.4-0.7) En\%), respectively. According to the European Food Safety Authority (EFSA), intake of EPA and DHA was below the recommended combined intake of $250 \mathrm{mg}$ of EPA plus DHA/d in 524 RTR (82\%), whereas intake of ALA was in accordance with the guidelines (0.5 En\%) in 352 RTR $(55 \%)^{(19)}$. Patient characteristics according to tertiles of EPA-DHA and ALA intakes (En\%) are shown in online Supplementary Tables S1 and S2, respectively.

The associations of baseline characteristics with intake of $n-3$ fatty acids are shown in Table 2 . Age was found to be positively associated with the marine-derived $n-3$ fatty acids $(\beta=0.094$, $P=0.02)$, whereas age was inversely associated with intake of plant-derived ALA $(\beta=-0.093, P=0.02)$. In addition, moderate alcohol intake $(\beta=0 \cdot 197, P<0 \cdot 001)$ and former smoking habits $(\beta=0 \cdot 128, P=0 \cdot 01)$ were positively associated with EPA-DHA intake, whereas there was no association with ALA intake. Furthermore, total fat intake $(\beta=-0.157, P<0.001)$ and total carbohydrate intake $(\beta=-0.272, \quad P<0.001)$ were inversely associated with EPA-DHA intake, whereas total fat intake $(\beta=0.318, P<0 \cdot 001)$ had a positive association with ALA intake. Carbohydrate intake was not associated with ALA intake. We found no significant interaction of $n$-3 fatty acid intake with potential explanatory variables for the association with graft failure and all-cause mortality.

For prospective analyses, the median follow-up was 3.1 (IQR $2 \cdot 7-3 \cdot 8)$ years. During the follow-up period, forty-one RTR developed graft failure, whereas sixty-seven RTR died.

Results for Cox regression analyses for graft failure are shown in Table 3. Neither EPA-DHA nor ALA intake appeared to be associated with graft failure (hazard ratio (HR) 0.83; $95 \%$ CI $0 \cdot 50,2 \cdot 27$; and HR 0.90; $95 \%$ CI 0.74, 1.09, respectively, per $0 \cdot 1$ En\% difference) while treating intakes as continuous variables. Findings were similar when intakes were treated as tertiles.

Results of Cox regression analyses for all-cause mortality are shown in Table 4. In age- and sex-adjusted analyses, with EPA-DHA intake treated as a continuous variable, there was no association with all-cause mortality (HR 0.79; 95\% CI 0.54,
Table 1. Baseline characteristics of 637 renal transplant recipients (Mean values and standard deviations; medians and interquartile ranges (IQR); numbers and percentages)

\begin{tabular}{|c|c|c|c|}
\hline Characteristics & Mean & SD & Range (min-max) \\
\hline \multicolumn{4}{|l|}{ Demographics } \\
\hline Age (years) & 53 & 13 & $18-78$ \\
\hline Sex (\% male) & \multicolumn{2}{|c|}{57} & \\
\hline BMI $\left(\mathrm{kg} / \mathrm{m}^{2}\right)$ & $26 \cdot 6$ & 4.8 & $15 \cdot 7-45 \cdot 0$ \\
\hline $\mathrm{BSA}\left(\mathrm{m}^{2}\right)$ & 1.9 & 0.2 & $1 \cdot 1-2 \cdot 8$ \\
\hline \multicolumn{4}{|l|}{ Smoking behaviour (\%) } \\
\hline No & \multicolumn{2}{|c|}{$41 \cdot 3$} & \\
\hline Former & \multicolumn{2}{|c|}{$45 \cdot 8$} & \\
\hline Current & \multicolumn{2}{|c|}{$12 \cdot 9$} & \\
\hline \multicolumn{4}{|l|}{ Diabetes (yes) } \\
\hline$n$ & \multirow{2}{*}{\multicolumn{2}{|c|}{$\begin{array}{c}154 \\
24 \cdot 2\end{array}$}} & \\
\hline$\%$ & & & \\
\hline \multicolumn{4}{|l|}{ Haemodynamic parameters } \\
\hline $\mathrm{SBP}(\mathrm{mmHg})$ & 136 & 17 & 80-200 \\
\hline $\mathrm{DBP}(\mathrm{mmHg})$ & 83 & 11 & $54-125$ \\
\hline MAP $(\mathrm{mmHg})$ & 101 & 12 & $66-145$ \\
\hline Heart rate $(\mathrm{bpm})$ & 69 & 12 & $41-122$ \\
\hline \multicolumn{4}{|l|}{ Medication use } \\
\hline Antihypertensives (\%) & \multicolumn{2}{|c|}{87.9} & \\
\hline Statins (\%) & \multicolumn{2}{|c|}{$52 \cdot 8$} & \\
\hline CNI (\%) & & & \\
\hline Proliferation inhibitors (\%) & & & \\
\hline mTor (\%) & & & \\
\hline Prednisolone dose $(\mathrm{mg} / \mathrm{d})$ & & & $0-20 \cdot 0$ \\
\hline Median & 1 & & \\
\hline IQR & & & \\
\hline Renal function parameters & & & \\
\hline Time after transplantation (years) & & & $0 \cdot 2-40$ \\
\hline Median & & & \\
\hline IQR & $1 \cdot 8-$ & $2 \cdot 1$ & \\
\hline Serum creatinine $(\mu \mathrm{mol} / \mathrm{l})$ & & & $50-591$ \\
\hline Median & 12 & & \\
\hline IQR & 100 & 160 & \\
\hline eGFR $\left(\mathrm{ml} / \mathrm{min}\right.$ per $\left.1.73 \mathrm{~m}^{2}\right)$ & 49 & 20 & $7-115$ \\
\hline Proteinuria $\geq 0.5 \mathrm{~g} / \mathrm{d}(\%)$ & & & \\
\hline$n$ & 13 & & \\
\hline$\%$ & 2 & & \\
\hline Protein excretion $(\mathrm{mg} / 24 \mathrm{~h})$ & & & $0.00-8.91$ \\
\hline Median & & 19 & \\
\hline IQR & 0.02 & 0.35 & \\
\hline Albumin excretion (mg/24h) & & & $0.02-6258$ \\
\hline Median & & & \\
\hline IQR & 10 & & \\
\hline Serum parameters & & & \\
\hline Albumin (g/l) & 43.0 & 3.0 & $28-51$ \\
\hline Cholesterol (mmol/l) & $5 \cdot 1$ & $1 \cdot 1$ & $2 \cdot 3-9 \cdot 7$ \\
\hline LDL-cholesterol $(\mathrm{mmol} / \mathrm{l})$ & 2.9 & 0.9 & $0.7-6.6$ \\
\hline HDL-cholesterol (mmol/li) & 1.4 & 0.5 & $0.4-3.5$ \\
\hline TAG $(\mathrm{mmol} / \mathrm{l})$ & & & $0.3-8.5$ \\
\hline Median & & & \\
\hline IQR & $1 \cdot 2$ & & \\
\hline $\mathrm{HbA1c}(\%)$ & $6 \cdot 0$ & 0.8 & $4.5-11 \cdot 8$ \\
\hline Hs-CRP (mg/l) & & & $0.1-114.0$ \\
\hline Median & & & \\
\hline IQR & & & \\
\hline Dietary intake (FFQ) & & & \\
\hline Energy intake & & & \\
\hline $\mathrm{kJ} / \mathrm{d}$ & 9117 & 2664 & $2770-27428$ \\
\hline $\mathrm{kcal} / \mathrm{d}$ & 2175 & 637 & $657-6575$ \\
\hline Total fat intake $(\mathrm{g} / \mathrm{d})$ & 88 & 34 & $21-412$ \\
\hline Total protein intake $(\mathrm{g} / \mathrm{d})$ & 82 & 20 & $25-177$ \\
\hline Total carbohydrates $(\mathrm{g} / \mathrm{d})$ & 249 & 78 & $16-539$ \\
\hline Alcohol intake (g/d) & & & $0.0-75 \cdot 8$ \\
\hline Median & & & \\
\hline IQR & 0.03 & $11 \cdot 2$ & \\
\hline$n-3$ Fatty acid intake & & & \\
\hline EPA-DHA intake $(\mathrm{mg} / \mathrm{d})$ & & & $0.0-1370$ \\
\hline Median & & & \\
\hline IQR & $28 \cdot 2-$ & $04 \cdot 8$ & \\
\hline
\end{tabular}


Table 1. Continued

\begin{tabular}{|c|c|c|c|}
\hline Characteristics & Mean & SD & Range (min-max) \\
\hline Relative amount (En\%) & \multirow{2}{*}{\multicolumn{2}{|c|}{0.04}} & \\
\hline ALA intake $(g / d)$ & & & $0.3-6.4$ \\
\hline Median & \multicolumn{2}{|c|}{$1 \cdot 2$} & \\
\hline IQR & \multicolumn{2}{|c|}{$0.9-1.7$} & \\
\hline Relative amount (En\%) & \multicolumn{2}{|c|}{0.5} & \\
\hline
\end{tabular}

BSA, body surface area; SBP, systolic blood pressure; DBP, diastolic blood pressure; MAP, mean arterial pressure; bpm, beats per minute; $\mathrm{CNI}$, calcineurin inhibitor; mTor, mammalian target of rapamycin; eGFR, estimated glomerular filtration rate; $\mathrm{HbA1c}$, glycated $\mathrm{Hb}$; Hs-CRP, high-sensitive C-reactive protein; En\%, energy percentage, ALA, $a$-linolenic acid.

Table 2. Association of baseline EPA-DHA and ALA intakes with baseline parameters (Regression coefficients $(\beta)$ per standard deviation of EPA-DHA and ALA intakes)

\begin{tabular}{|c|c|c|c|c|}
\hline \multirow[b]{2}{*}{ Dependent variables } & \multicolumn{2}{|c|}{ EPA-DHA } & \multicolumn{2}{|c|}{ ALA } \\
\hline & $\beta$ & $P$ & $\beta$ & $P$ \\
\hline \multicolumn{5}{|l|}{ Demographics } \\
\hline Age (years) & 0.094 & 0.02 & -0.093 & 0.02 \\
\hline Sex & 0.033 & 0.41 & -0.068 & 0.09 \\
\hline BMI $\left(\mathrm{kg} / \mathrm{m}^{2}\right)$ & 0.068 & 0.09 & 0.065 & 0.10 \\
\hline $\operatorname{BSA}\left(\mathrm{m}^{2}\right)$ & -0.025 & 0.53 & 0.095 & 0.02 \\
\hline \multicolumn{5}{|l|}{ Smoking habits } \\
\hline Former & 0.128 & 0.003 & -0.017 & 0.69 \\
\hline Current & 0.062 & 0.15 & -0.061 & 0.16 \\
\hline Diabetes (\% yes) & 0.005 & 0.90 & -0.002 & 0.96 \\
\hline \multicolumn{5}{|l|}{ Haemodynamic factors } \\
\hline $\mathrm{SBP}(\mathrm{mmHg})$ & -0.045 & 0.26 & -0.032 & 0.42 \\
\hline $\mathrm{DBP}(\mathrm{mmHg})$ & 0.002 & 0.96 & 0.073 & 0.07 \\
\hline MAP $(\mathrm{mmHg})$ & -0.036 & 0.37 & 0.005 & 0.89 \\
\hline Heart rate (bpm) & 0.063 & 0.12 & 0.061 & 0.14 \\
\hline \multicolumn{5}{|l|}{ Dietary intake (FFQ) } \\
\hline Total fat intake $(\mathrm{g} / \mathrm{d})$ & -0.157 & $<0.001$ & 0.318 & $<0.001$ \\
\hline Total protein intake $(\mathrm{g} / \mathrm{d})$ & -0.035 & 0.38 & 0.066 & 0.09 \\
\hline Total carbohydrates $(\mathrm{g} / \mathrm{d})$ & -0.273 & $<0.001$ & 0.009 & 0.83 \\
\hline \multicolumn{5}{|l|}{ Alcohol intake (g/d) } \\
\hline $0-10$ & 0.116 & 0.05 & 0.149 & 0.01 \\
\hline 10-30 & 0.197 & 0.001 & 0.064 & 0.27 \\
\hline$>30$ & 0.113 & 0.01 & 0.048 & 0.29 \\
\hline \multicolumn{5}{|l|}{ Medication use } \\
\hline Antihypertensives (\% yes) & -0.015 & 0.70 & 0.019 & 0.63 \\
\hline Statins (\% yes) & 0.045 & 0.26 & -0.062 & 0.12 \\
\hline \multicolumn{5}{|l|}{ Renal function factors } \\
\hline Serum creatinine $(\mu \mathrm{mol} / \mathrm{l})$ & 0.021 & 0.59 & 0.004 & 0.91 \\
\hline eGFR $\left(\mathrm{ml} / \mathrm{min}\right.$ per $\left.1.73 \mathrm{~m}^{2}\right)$ & -0.063 & 0.11 & 0.039 & 0.33 \\
\hline Albuminuria* (mg/24h) & 0.008 & 0.84 & -0.082 & 0.04 \\
\hline Proteinuria* $(\geq 0.5 \mathrm{~g} / \mathrm{d})^{\prime}$ & -0.004 & 0.91 & -0.072 & 0.07 \\
\hline \multicolumn{5}{|l|}{ Serum parameters } \\
\hline Albumin (g/l) & -0.022 & 0.58 & 0.011 & 0.79 \\
\hline Cholesterol (mmol/l) & 0.048 & 0.23 & -0.022 & 0.58 \\
\hline TAG (mmol/l) & 0.004 & 0.91 & 0.002 & 0.95 \\
\hline Hs-CRP $(\mathrm{mg} / \mathrm{l})^{*}$ & 0.068 & 0.10 & 0.052 & 0.21 \\
\hline HbA1c (\%) & 0.042 & 0.30 & 0.007 & 0.86 \\
\hline
\end{tabular}

BSA, body surface area; SBP, systolic blood pressure; DBP, diastolic blood pressure; MAP, mean arterial pressure; bpm, beats per minute; eGFR, estimated glomerular filtration rate; Hs-CRP, high-sensitive C-reactive protein; $\mathrm{HbA} 1 \mathrm{c}$, glycated haemoglobin; ALA, $a$-linolenic acid.

${ }^{*}$ Log-transformed for analysis.

1.15 per $0 \cdot 1$ En\% difference), whereas ALA intake was significantly associated with increased all-cause mortality risk ( $\mathrm{HR}=1 \cdot 17 ; 95 \% \mathrm{CI} 1 \cdot 04,1.31$ per $0 \cdot 1 \mathrm{En} \%$ difference) (model 1) (Fig. 1(a)). Moreover, RTR in the highest tertile of ALA intake
( $>0.60 \mathrm{En} \%$ ) were at 2-fold higher risk of all-cause mortality $(\mathrm{HR}=2.21 ; 95 \%$ CI $1.23,3.97)$ than those in the lowest tertile (reference tertile) $(<0.45$ En\%). Associations remained materially unchanged after cumulative adjustment for potential explanatory variables, including BMI, DBP, alcohol intake, smoking behaviour, fat, protein and carbohydrate intake, and proteinuria.

In secondary analyses, the association of ALA intake with all-cause mortality was analysed using g/d instead of En\%. In age- and sex-adjusted analyses, RTR in the highest tertile of ALA intake $(>1.45 \mathrm{~g} / \mathrm{d})$ were at almost 2-fold higher risk of all-cause mortality (HR 1.90; 95\% CI 1.07, 3.37) compared with the lowest tertile (reference tertile) $(<1 \cdot 14 \mathrm{~g} / \mathrm{d})$. Our findings did not change after further cumulative adjustment for potential explanatory variables. We also calculated the intake of ALA as a proportion of the total intake of fatty acids. It remained that there was no association of ALA intake (now expressed as proportion of total fatty acid intake) with graft failure (HR 0.93; 95\% CI 0.80, 1.08 per $0.1 \%$ difference). It also remained that there was an association of ALA intake (now expressed as proportion of total fatty acid intake) with increased risk for mortality (HR 1.14; $95 \%$ CI $1.02,1.27$ per $0.1 \%$ difference, $P=0.02$ ).

In sensitivity analyses, we excluded patients with extreme intake of $n$ - 3 fatty acids. HR of EPA-DHA and ALA intakes for graft failure and all-cause mortality remained materially unchanged, with no associations for graft failure, and $\mathrm{HR}=0.78$ (95\% CI 0.50 , $1 \cdot 22)$ and $\mathrm{HR}=1 \cdot 21$ (95\% CI 1.06, 1.37) per $0 \cdot 1 \mathrm{En} \%$ difference for the associations of EPA-DHA and ALA intake with all-cause mortality, respectively (Fig. 1(b)).

\section{Discussion and conclusion}

Our study is, to the best of our knowledge, the first to separately investigate the associations of the marine-derived $n-3$ fatty acids EPA and DHA and the plant-derived $n-3$ fatty acid ALA with long-term outcome in a large cohort of RTR. Both EPA-DHA and ALA intakes were not associated with graft failure. In addition, EPA-DHA intake was not associated with all-cause mortality, whereas ALA intake was positively associated with all-cause mortality.

The $n$ - 3 fatty acids EPA, DHA and ALA are long-chain PUFA. EPA and DHA are mainly found in fatty fish, whereas vegetable oils such as linseed, soyabean and rapeseed oils are the main sources of ALA ${ }^{(20,21)}$. According to the EFSA, the combined intake of EPA plus DHA was found to be below the recommended intake in $82 \%$ of RTR, while $45 \%$ had an inadequate intake of ALA. Various recommendations for PUFA intakes are circulating around the world ${ }^{(22)}$. For instance, the ALA intake recommendation in Australia is $2.0 \mathrm{~g} / \mathrm{d}$ ( $1 \mathrm{En} \%$ ), whereas in the USA and Canada the recommended ALA intake is $1.35 \mathrm{~g} / \mathrm{d}(0.7 \mathrm{En} \%)^{(23)}$. According to these international guidelines, the number of RTR with an ALA intake below the recommended daily intake increases enormously.

At least two meta-analyses of randomised-controlled trials have investigated whether fish oil supplementation may improve renal function, rejection rates, and patient and graft survival in $\mathrm{RTR}^{(7,24)}$. Both these meta-analyses reported that 
Table 3. Cox regression analyses for prediction of graft failure based on $n-3$ fatty acid intakes (En\%) (Hazard ratios (HR) and $95 \%$ confidence intervals)

\begin{tabular}{|c|c|c|c|c|c|c|c|c|c|c|}
\hline \multirow[b]{3}{*}{ Models* } & \multicolumn{7}{|c|}{ Tertiles of $n-3$ fatty acid intake (En\%) } & & & \\
\hline & \multirow{2}{*}{$\frac{1}{\text { Ref. }}$} & \multicolumn{3}{|c|}{ II } & \multicolumn{3}{|c|}{ III } & \multicolumn{3}{|c|}{ Continuous (En\% per 0.1 En\% difference) } \\
\hline & & $\mathrm{HR}$ & $95 \% \mathrm{Cl}$ & $P$ & $\mathrm{HR}$ & $95 \% \mathrm{Cl}$ & $P$ & $\mathrm{HR}$ & $95 \% \mathrm{Cl}$ & $P$ \\
\hline \multicolumn{11}{|c|}{ EPA-DHA intake } \\
\hline 1 & 1.00 & 1.08 & $0.53,2 \cdot 20$ & 0.84 & 0.79 & $0.36,1.73$ & 0.56 & 0.83 & $0.50,2.27$ & 0.46 \\
\hline 2 & 1.00 & 1.09 & $0.53,2.24$ & 0.81 & 0.81 & $0.37,1.77$ & 0.59 & 0.98 & $0.92,1.05$ & 0.53 \\
\hline 3 & 1.00 & 1.29 & $0.61,2.72$ & 0.51 & 0.95 & $0.42,2.13$ & 0.89 & 0.92 & $0.56,1.49$ & 0.72 \\
\hline 4 & 1.00 & $1 \cdot 27$ & $0.60,2.69$ & 0.54 & 0.91 & $0.40,2 \cdot 11$ & 0.83 & 0.89 & $0.53,1.49$ & 0.65 \\
\hline 5 & 1.00 & 1.02 & $0.47,1.23$ & 0.96 & 0.72 & $0.30,1.72$ & 0.45 & 0.77 & $0.43,1.39$ & 0.39 \\
\hline \multicolumn{11}{|c|}{ ALA intake } \\
\hline 1 & 1.00 & 0.97 & $0.45,2.09$ & 0.95 & $1 \cdot 10$ & $0.51,2.36$ & 0.81 & 0.90 & $0.74,1.09$ & 0.27 \\
\hline 2 & 1.00 & 0.99 & $0.46,2 \cdot 12$ & 0.98 & $1 \cdot 13$ & $0.52,2.44$ & 0.75 & 0.90 & $0.75,1.09$ & 0.29 \\
\hline 3 & 1.00 & 1.26 & $0.56,2.83$ & 0.58 & 1.47 & $0.65,3.28$ & 0.35 & 0.95 & $0.78,1.14$ & 0.56 \\
\hline 4 & 1.00 & 1.34 & $0.58,3.08$ & 0.49 & 1.79 & $0.73,4.39$ & 0.20 & 0.96 & $0.77,1.19$ & 0.70 \\
\hline 5 & 1.00 & $1 \cdot 16$ & $0.47,2.85$ & 0.75 & 1.99 & $0.80,4.98$ & 0.14 & 1.02 & $0.81,1.30$ & 0.86 \\
\hline
\end{tabular}

En\%, energy percentage; Ref., referent values; ALA, a-linolenic acid.

* Model 1, adjusted for age and sex; model 2, as model 1, additionally adjusted for BMI; model 3, as model 2, additionally adjusted for alcohol intake and smoking habits; model 4, as model 3 , additionally adjusted for fat, protein and carbohydrate intakes; model 5 , as model 4 , additionally adjusted for proteinuria and diastolic blood pressure. Proteinuria was log-transformed for analyses.

Table 4. Cox regression analyses for the prediction of all-cause mortality based on $n-3$ fatty acid intakes (En\%) (Hazard ratios (HR) and $95 \%$ confidence intervals)

\begin{tabular}{|c|c|c|c|c|c|c|c|c|c|c|}
\hline \multirow[b]{3}{*}{ Models* } & \multicolumn{7}{|c|}{ Tertiles of $n-3$ fatty acid intake (En\%) } & & & \\
\hline & \multirow{2}{*}{$\frac{1}{\text { Ref. }}$} & \multicolumn{3}{|c|}{ II } & \multicolumn{3}{|c|}{ III } & \multicolumn{3}{|c|}{ Continuous (En\% per 0.1 En\% difference) } \\
\hline & & $\mathrm{HR}$ & $95 \% \mathrm{Cl}$ & $P$ & $\mathrm{HR}$ & $95 \% \mathrm{Cl}$ & $P$ & $\mathrm{HR}$ & $95 \% \mathrm{Cl}$ & $P$ \\
\hline \multicolumn{11}{|c|}{ EPA-DHA intake } \\
\hline 1 & 1.00 & $1 \cdot 25$ & $0.71,2 \cdot 20$ & 0.44 & 0.72 & $0.39,1.35$ & 0.31 & 0.79 & $0.54,1.15$ & 0.22 \\
\hline 2 & 1.00 & 1.35 & $0.71,2 \cdot 20$ & 0.44 & 0.72 & $0.39,1.35$ & 0.31 & 0.79 & $0.54,1.16$ & 0.23 \\
\hline 3 & 1.00 & 1.41 & $0.77,2.54$ & 0.26 & 0.89 & $0.46,1.71$ & 0.71 & 0.88 & $0.61,1.29$ & 0.52 \\
\hline 4 & 1.00 & 1.56 & $0.86,2.86$ & 0.15 & 1.02 & $0.52,2.00$ & 0.97 & 0.95 & $0.64,1.40$ & 0.79 \\
\hline 5 & 1.00 & 1.56 & $0.85,2.86$ & 0.15 & 0.96 & $0.48,1.93$ & 0.91 & 0.90 & $0.60,1.34$ & 0.60 \\
\hline \multicolumn{11}{|c|}{ ALA intake } \\
\hline 1 & 1.00 & 1.06 & $0.55,2.07$ & 0.86 & $2 \cdot 21$ & $1 \cdot 23,3.97$ & 0.01 & $1 \cdot 17$ & $1.04,1.32$ & 0.01 \\
\hline 2 & 1.00 & 1.07 & $0.55,2.07$ & 0.85 & $2 \cdot 22$ & $1.24,3.98$ & 0.01 & $1 \cdot 17$ & $1.04,1.32$ & 0.01 \\
\hline 3 & 1.00 & $1 \cdot 13$ & $0.56,2.25$ & 0.74 & $2 \cdot 20$ & $1 \cdot 20,4.03$ & 0.01 & $1 \cdot 17$ & $1.03,1.32$ & 0.02 \\
\hline 4 & 1.00 & 1.05 & $0.52,2 \cdot 14$ & 0.88 & 2.08 & $1.07,4.05$ & 0.03 & $1 \cdot 16$ & $1.01,1.35$ & 0.04 \\
\hline 5 & 1.00 & 0.92 & $0.45,1.90$ & 0.83 & 2.04 & $1.05,3.98$ & 0.04 & $1 \cdot 19$ & $1.02,1.38$ & 0.03 \\
\hline
\end{tabular}

En\%, energy percentage; Ref., referent values; ALA, $a$-linolenic acid.

* Model 1, adjusted for age and sex; model 2, as model 1, additionally adjusted for BMI; model 3, as model 2, additionally adjusted for alcohol intake and smoking habits; model 4, as model 3 , additionally adjusted for fat, protein and carbohydrate intakes; model 5 , as model 4 , additionally adjusted for proteinuria and diastolic blood pressure. Proteinuria was log-transformed for analyses.

there was insufficient evidence to conclude that fish oil has a beneficial effect on either of these outcomes. It was mentioned that the meta-analyses were hampered by methodological problems, including different doses of fish oils, different duration and timing of treatment, studies with small numbers of patients, inadequate randomisation and lack of blinding ${ }^{(7)}$. Recently, two studies in a large observational cohort, with 569 allograft failures and 406 deaths in 1990 RTR that were followed-up for 6.8 years, showed significant inverse associations of plasma phospholipid concentrations of marinederived $n-3$ fatty acids with graft failure and mortality in $\mathrm{RTR}^{(25,26)}$. Interestingly, in a large systematic review of the effects of $n-3$ fatty acids on CVD in the general population it was concluded that EPA-DHA intake reduced the rates of all-cause mortality, cardiac and sudden death and possibly stroke $^{(8)}$. These authors analysed ALA separately from EPA-DHA and found that ALA was not associated with these positive findings ${ }^{(7,8)}$. In another systematic review, it was concluded that supplementation of EPA and DHA or fish oil has a consistent beneficial effects on serum lipids, whereas this is not the case for supplementation with ALA ${ }^{(27)}$. This pattern of decrease in risk associated with high intake of EPA-DHA and increase in risk associated with high intake of ALA are also present in studies including biomarkers. A recent study in which circulating levels of EPA, DHA and ALA were measured in 60-year-old adults showed that high circulating concentrations of EPA and DHA were found to be associated with low risk of all-cause mortality, whereas it was found that there was a trend 

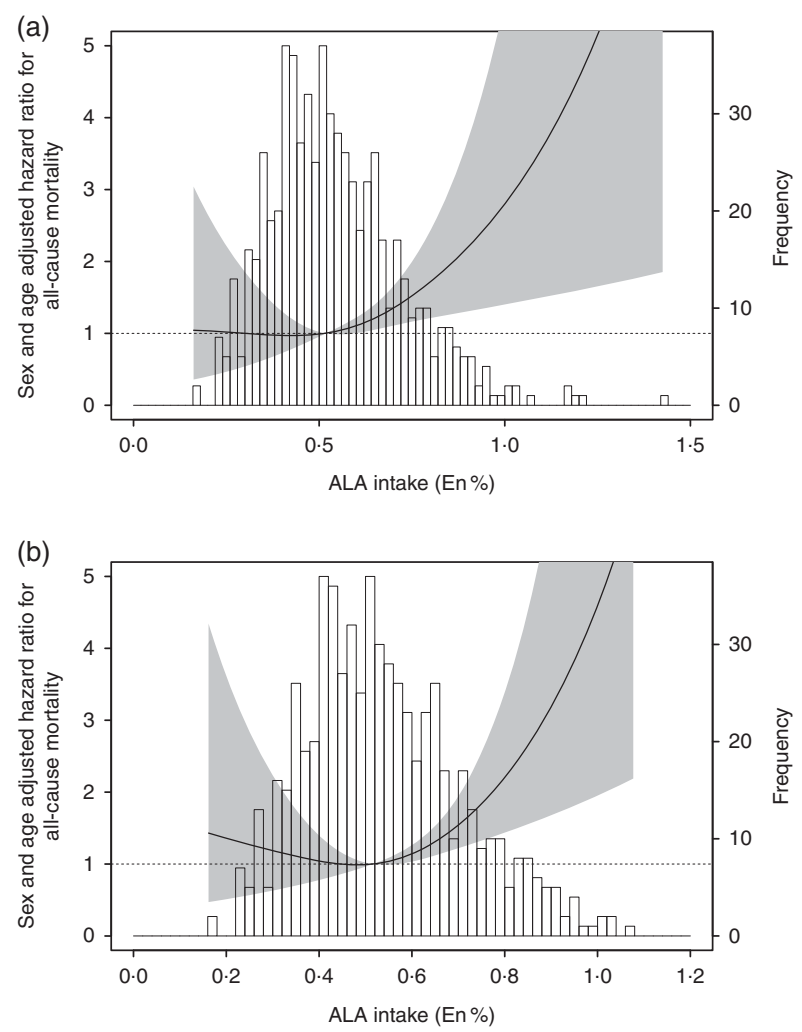

Fig. 1. (a) Restricted cubic spline showing the association between $a$-linolenic acid (ALA) intake (En\%) and all-cause mortality in 637 renal transplant recipients (RTR). (b) Restricted cubic spline showing the association between ALA intake (En\%) and all-cause mortality in 632 RTR. En\%, energy percentage. —, Age- and sex-adjusted risk for all-cause mortality; $\square, 95 \% \mathrm{Cl}$ of the $\mathrm{HR}$.

towards an association of high circulating levels of ALA with an increased risk for all-cause mortality ${ }^{(28)}$.

In our cohort, there was no association of EPA-DHA intake with all-cause mortality, whereas a high-ALA intake was associated with a significantly increased risk of all-cause mortality. The fact that the association between the marine-derived $n-3$ fatty acid intakes and all-cause mortality did not reach significance may have been the consequence of the relatively small number of cases (sixty-seven deaths in a cohort of 637 RTR during a follow-up period of 3.1 years). Indeed, in a recent analysis of a cohort study, with 406 deaths in 1990 RTR followed-up for 6.8 years, it was found that circulating levels of marine $n-3$ fatty acid in plasma phospholipids were inversely associated with all-cause and cardiovascular mortality after renal transplantation ${ }^{(26)}$. Other than in size and duration of follow-up, this study differed from our study with respect to percentage of males ( $67 \%$ in the study by Eide et al. $v .57 \%$ in our study), percentage of diabetics (18.2 v. 24.2\%), percentage of former smokers $(36.2 v .45 \cdot 8 \%)$ and eGFR (57v $49 \mathrm{ml} / \mathrm{min}$ per $\left.1.73 \mathrm{~m}^{2}\right)$, whereas age (52v.53 years), BMI (24.8v. $26.6 \mathrm{~kg} / \mathrm{m}^{2}$ ) and percentage of current smokers (16.0 v. 12.9\%) were less dissimilar. Another difference between the studies is the way in which $n-3$ fatty acid exposure was assessed (i.e. a status marker ( $n-3$ fatty acids in plasma phospholipids) in the study by Eide et al. $v$. intake (FFQ) in our study).

Results regarding the effect of EPA-DHA and ALA intake on graft failure are inconsistent. As mentioned earlier, meta-analyses of randomised controlled trials on this subject found no effect of EPA-DHA on graft failure ${ }^{(7,24)}$, which is in line with our finding of a virtual absence of an association of EPA-DHA and ALA intakes with graft failure in RTR. An intervention study in 2344 postmyocardial infarction patients suggests, however, that a combined intake of $400 \mathrm{mg} / \mathrm{d}$ of EPA and DHA provides a small beneficial effect on renal function, whereas this was not the case for ALA ${ }^{(29)}$. A positive effect of EPA-DHA was also suggested by a study of circulating levels of EPA and DHA. In an observational cohort study from Norway, it was described that high levels of marinederived $n-3$ fatty acids in plasma phospholipids were associated with better allograft survival. The authors showed an $11 \%$ reduction in risk of graft loss for every $1.0 \mathrm{wt} \%$ increase in EPA-DHA levels ${ }^{(25)}$.

Recently, two authoritative meta-analyses in the field of $n-3$ fatty acid intake and mortality investigated whether there is a threshold for the relation of EPA-DHA intake with outcome $^{(30,31)}$. These studies found that there was a decline in $\mathrm{CHD}^{(30,31)}$ and total mortality ${ }^{(31)}$ with increasing EPA-DHA intake in the range of EPA-DHA intake from 0 to $250 \mathrm{mg} / \mathrm{d}$, whereas there was no further decline in CHD and total mortality with increasing intake beyond $250 \mathrm{mg} / \mathrm{d}$. In our cohort, the median EPA-DHA intake was 92.1 (IQR 28.2-204.8) $\mathrm{mg} / \mathrm{d}$, with only $18 \%$ of the population consuming $>250 \mathrm{mg}$ of EPA-DHA/d. Therefore, the EPA-DHA intake in our cohort was rather low compared with the threshold determined in the previous meta-analyses.

The reason why a higher ALA intake is associated with increased risk of all-cause mortality in RTR is unknown. ALA can be metabolised via at least two pathways. First, ALA is a preferred substrate for $\beta$-oxidation, with either complete oxidation to $\mathrm{CO}_{2}$ and water or incomplete conversion to intermediates that may subsequently be used for the synthesis of fatty acids and cholesterol ${ }^{(32,33)}$.

A second route is by conversion of ALA into EPA and subsequently into $\mathrm{DHA}^{(32,34)}$. Rate limiting steps in this pathway include the activities of $\Delta 5$ and $\Delta 6$ desaturases ${ }^{(35,36)}$. In RTR, this route, but also $\beta$-oxidation, could be affected by glucocorticoids, a class of immunosuppressive drugs used to protect the transplanted kidney from rejection ${ }^{(37,38)}$. In our cohort, $99 \%$ of the RTR were treated with prednisolone. Glucocorticoids can depress the activities of $\Delta 5$ and $\Delta 6$ desaturases, giving rise to altered fatty acid precursor/metabolic ratios, possibly including the ALA:EPA-DHA ratio ${ }^{(35,39)}$.

Finally, it is also possible that the association of ALA intake with high mortality risk might not be through the intake of ALA itself but rather via its decomposed products produced during baking or frying of ALA-rich foods ${ }^{(40)}$. ALA can be found in various widely used vegetable oils and is highly susceptible to peroxidation during heating ${ }^{(20,40)}$. The degraded products are hazardous to health and adverse effects may possibly become amplified in susceptible patients, such as RTR ${ }^{(40)}$.

Strengths of the present study include its large population size. In total, data of 637 RTR were used for analyses, which made the results reliable. Another strength of the present study is the prospective analyses carried out with Cox regression in RTR using 3-year follow-up information of patients. At present, studies on the long-term effects of $n$ - 3 fatty acids are especially 
carried out in the general population. In our study, we were able to identify specific long-term association in our target group that nevertheless needs further investigations of causality. The third strong point is the inclusion of intake of ALA in the prospective study. Most studies in the field of $n$-3 fatty acids predominantly focus on EPA-DHA intake, whereas ALA intake might be important as well. We analysed EPA-DHA and ALA intakes separately, to investigate the specific effects of the marine-derived and plant-derived $n-3$ fatty acids, and found a difference between the two.

We acknowledge that our study also has some limitations. The present study is an observational epidemiological study, which may only suggest biological significance. Another limitation of our study is the use of an FFQ for measuring dietary intake. Measurements of intake were based on self-report, which may lead to misclassification, under-reporting and over-reporting. Furthermore, intakes were only measured once at baseline, whereas changes in intake and clinical parameters may appear during the 3 years of follow-up. Finally, dietary intake and status form complementary information on $n-3$ fatty acid exposure. The present study made use of dietary $n-3$ fatty acid intake, but not status. Future studies should therefore focus on $n$ - 3 fatty acid biomarkers also.

In conclusion, in RTR, EPA-DHA and ALA intakes showed no clear association with graft failure. EPA-DHA intake showed no association with all-cause mortality, whereas ALA intake was associated with increased all-cause mortality. Although the association between ALA intake and all-cause mortality was strong, a causal relationship in RTR is as yet questionable. Further investigation on the long-term outcome of $n$ - 3 fatty acids, particularly ALA, in RTR is warranted.

\section{Acknowledgements}

The funder of the present study was the Top Institute Food and Nutrition, which is a public-private partnership that generates vision on scientific breakthroughs in food and nutrition, resulting in the development of innovative products and technologies. Partners are major Dutch food companies and research organisations. Top Institute Food and Nutrition had no role in the design, analysis or writing of this article, but provided financial support (A-1003). The cohort on which the study was based is registered at clinicaltrials.gov as 'TransplantLines Food and Nutrition Biobank and Cohort Study (TxL-FN)' with number NCT02811835.

I. G. P. analysed the data and drafted the manuscript. E. G. G. helped with analysing the data and participated in article revisions. E. v. d. B. was responsible for the study design, the concept and acquisition of the data. S. S. S.-M. and F. A. J. M. participated in intellectual contributions and article revisions. G. N. was responsible for the study design and concept and participated in article revisions. R. O. B. G., I. P. K. and M. M. J. participated in article revisions. S. J. L. B. initiated the study, was responsible for the study design and concept, and edited the article. All the authors gave final approval of the manuscript to be published.

The authors declare that there are no conflicts of interest.

\section{Supplementary material}

For supplementary material/s referred to in this article, please visit https://doi.org/10.1017/S0007114516004207

\section{References}

1. Lysaght MJ (2002) Maintenance dialysis population dynamics: current trends and long-term implications. J Am Soc Nephrol 13, Suppl. 1, S37-S40.

2. Levey AS \& Coresh J (2012) Chronic kidney disease. Lancet 379, 165-180.

3. Wolfe RA, Ashby VB, Milford EL, et al. (1999) Comparison of mortality in all patients on dialysis, patients on dialysis awaiting transplantation, and recipients of a first cadaveric transplant. $N$ Engl J Med 341, 1725-1730.

4. Schippers HM \& Kalff MW (1976) Cost comparison haemodialysis and renal transplantation. Tissue Antigens 7, 86-90.

5. Merville P (2005) Combating chronic renal allograft dysfunction: optimal immunosuppressive regimens. Drugs 65, 615-631.

6. Hariharan S, Johnson CP, Bresnahan BA, et al. (2000) Improved graft survival after renal transplantation in the United States, 1988 to 1996. N Engl J Med 342, 605-612.

7. Lim AK, Manley KJ, Roberts MA, et al. (2007) Fish oil treatment for kidney transplant recipients: a meta-analysis of randomized controlled trials. Transplantation 83, 831-838.

8. Wang C, Harris WS, Chung M, et al. (2006) n-3 Fatty acids from fish or fish-oil supplements, but not alpha-linolenic acid, benefit cardiovascular disease outcomes in primary- and secondary-prevention studies: a systematic review. Am J Clin Nutr 84, 5-17.

9. Warensjo E, Sundstrom J, Vessby B, et al. (2008) Markers of dietary fat quality and fatty acid desaturation as predictors of total and cardiovascular mortality: a population-based prospective study. Am J Clin Nutr 88, 203-209.

10. van den Berg E, Engberink MF, Brink EJ, et al. (2013) Dietary protein, blood pressure and renal function in renal transplant recipients. Br J Nutr 109, 1463-1470.

11. Feunekes GI, Van Staveren WA, De Vries JH, et al. (1993) Relative and biomarker-based validity of a food-frequency questionnaire estimating intake of fats and cholesterol. $\mathrm{Am} \mathrm{J}$ Clin Nutr 58, 489-496.

12. Stichting NEVO (2006) Nederlands voedingsstoffen bestand: NEVO tabel 2006 (Dutch Nutrient Databank: NEVO table 2006). The Hague: Voorlichtingsbureau voor de voeding.

13. Abbasi A, Peelen LM, Corpeleijn E, et al. (2012) Prediction models for risk of developing type 2 diabetes: systematic literature search and independent external validation study. BMJ 345, e5900.

14. van den Berg E, Geleijnse JM, Brink EJ, et al. (2012) Sodium intake and blood pressure in renal transplant recipients. Nephrol Dial Transplant 27, 3352-3359.

15. Levey AS, Stevens LA, Schmid CH, et al. (2009) A new equation to estimate glomerular filtration rate. Ann Intern Med 150, 604-612.

16. Perneger TV (1998) What's wrong with Bonferroni adjustments. BMJ 316, 1236-1238.

17. Redmond N, Booth JN 3rd, Tanner RM, et al. (2016) Prevalence of masked hypertension and its association with subclinical cardiovascular disease in African Americans: results from the Jackson Heart Study. J Am Heart Assoc 4, e002284.

18. Willett WC, Howe GR \& Kushi LH (1997) Adjustment for total energy intake in epidemiologic studies. Am J Clin Nutr $\mathbf{6 5}$, Suppl., 1220S-1228S; discussion 1229S-1231S.

19. European Food Safety Authority Panel on Dietetic Products \& Nutrition and Allergies Panel (2010) Scientific opinion on 
dietary reference values for fats, including saturated fatty acids, polyunsaturated fatty acids, monounsaturated fatty acids, trans fatty acids, and cholesterol. EFSA J 8, 107.

20. Kris-Etherton PM, Taylor DS, Yu-Poth S, et al. (2000) Polyunsaturated fatty acids in the food chain in the United States. Am J Clin Nutr 71, Suppl., 179S-188S.

21. Gebauer SK, Psota TL, Harris WS, et al. (2006) n-3 Fatty acid dietary recommendations and food sources to achieve essentiality and cardiovascular benefits. Am J Clin Nutr $\mathbf{8 3}$, Suppl., 1526S-1535S.

22. Meyer BJ, Mann NJ, Lewis JL, et al. (2003) Dietary intakes and food sources of omega- 6 and omega- 3 polyunsaturated fatty acids. Lipids 38, 391-398.

23. International Society for the Study of Fatty Acids and Lipids (2004) Recommendations for dietary intake of polyunsaturated fatty acids in healthy adults, June. http://www.issfal.org/newslinks/resources/publications/PUFAIntakeReccomdFinalReport. pdf (accessed January 2016).

24. Tatsioni A, Chung M, Sun Y, et al. (2005) Effects of fish oil supplementation on kidney transplantation: a systematic review and meta-analysis of randomized, controlled trials. J Am Soc Nephrol 16, 2462-2470.

25. Eide IA, Jenssen T, Hartmann A, et al. (2016) Plasma levels of marine $n-3$ polyunsaturated fatty acids and renal allograft survival. Nephrol Dial Transplant 31, 160-167.

26. Eide IA, Jenssen T, Hartmann A, et al. (2015) The association between marine $n-3$ polyunsaturated fatty acid levels and survival after renal transplantation. Clin J Am Soc Nephrol 10, 1246-1256.

27. Balk EM, Lichtenstein AH, Chung M, et al. (2006) Effects of omega-3 fatty acids on serum markers of cardiovascular disease risk: a systematic review. Atherosclerosis 189, 19-30.

28. Marklund M, Leander K, Vikstrom M, et al. (2015) Polyunsaturated fat intake estimated by circulating biomarkers and risk of cardiovascular disease and all-cause mortality in a populationbased cohort of 60-year-old men and women. Circulation $\mathbf{1 3 2}$, 586-594
29. Hoogeveen EK, Geleijnse JM, Kromhout D, et al. (2014) Effect of omega-3 fatty acids on kidney function after myocardial infarction: the alpha omega trial. Clin J Am Soc Nephrol 9, 1676-1683.

30. Harris WS, Mozaffarian D, Lefevre M, et al. (2009) Towards establishing dietary reference intakes for eicosapentaenoic and docosahexaenoic acids. J Nutr 139, 804S-819S.

31. Mozaffarian D \& Rimm EB (2006) Fish intake, contaminants, and human health: evaluating the risks and the benefits. JAMA 296, 1885-1899.

32. Burdge GC \& Wootton SA (2003) Conversion of alphalinolenic acid to palmitic, palmitoleic, stearic and oleic acids in men and women. Prostaglandins Leukot Essent Fatty Acids 69, 283-290

33. Cunnane SC, Ryan MA, Nadeau CR, et al. (2003) Why is carbon from some polyunsaturates extensively recycled into lipid synthesis? Lipids 38, 477-484.

34. Burdge GC (2006) Metabolism of alpha-linolenic acid in humans. Prostaglandins Leukot Essent Fatty Acids $\mathbf{7 5}$, 161-168.

35. Brenner RR (2003) Hormonal modulation of delta 6 and delta5 desaturases: case of diabetes. Prostaglandins Leukot Essent Fatty Acids 68, 151-162.

36. Nakamura MT \& Nara TY (2004) Structure, function, and dietary regulation of delta6, delta5, and delta9 desaturases. Annu Rev Nutr 24, 345-376.

37. Halloran PF, Melk A \& Barth C (1999) Rethinking chronic allograft nephropathy: the concept of accelerated senescence. J Am Soc Nephrol 10, 167-181.

38. Steiner RW \& Awdishu L (2011) Steroids in kidney transplant patients. Semin Immunopathol 33, 157.

39. de Alaniz MJ \& Marra CA (2003) Steroid hormones and fatty acid desaturases. Prostaglandins Leukot Essent Fatty Acids 68 , 163-170.

40. Ng CY, Leong XF, Masbah N, et al. (2014) Heated vegetable oils and cardiovascular disease risk factors. Vascul Pharmacol 61, 1-9. 